LIBRARY SIGLA

A-Wn Vienna, Österreichische Nationalbibliothek

D-AN Ansbach, Staatliche Bibliothek Ansbach

I-Fn Florence, Biblioteca Nazionale Centrale

I-Gu Genoa, Biblioteca Universitaria

I-Nc Naples, Conservatorio di Musica S. Pietro a Majella, Biblioteca

I-Rn Rome, Biblioteca Nazionale Centrale Vittorio Emanuele II

I-Vas Venice, Archivio di Stato

I-Vmc Venice, Museo Civico Correr, Biblioteca d'Arte e Storia Veneziana

I-Vnm Venice, Biblioteca Nazionale Marciana

\title{
OTHER ABBREVIATIONS
}

AM Analecta musicologica

COJ Cambridge Opera Journal

EM Early Music

EMH Early Music History

FI Forum Italicum

JAMS Journal of the American Musicological Society

JM Journal of Musicology

JRMA Journal of the Royal Musical Association

ML Music E Letters

RIM Rivista italiana di musicologia

$R Q \quad$ Renaissance Quarterly

SM Studi musicali 
This page intentionally left blank 